\title{
Antibacterial properties of in situ and surface functionalized impregnation of silver sulfadiazine in polyacrylonitrile nanofiber mats
}

This article was published in the following Dove Medical Press journal: International Journal of Nanomedicine

\author{
Sana Ullah' \\ Motahira Hashmi' \\ Davood Kharaghani' \\ Muhammad Qamar Khan' \\ Yusuke Saito' \\ Takayuki Yamamoto' \\ Jungsoon $\mathrm{Lee}^{2}$ \\ Ick Soo Kim' \\ 'Nano Fusion Technology Research \\ Group, Institute of Frontier Fiber \\ Engineering, Shinshu University, \\ Ueda, Nagano, Japan; ${ }^{2}$ Department \\ of Clothing and Textiles, College of \\ Human Ecology, Chungnam University, \\ Daejeon, South Korea
}

Background: Silver, incorporation with natural or synthetic polymers, has been used as an effective antibacterial agent since decades. Silver has potential applications in healthcare especially in nanoparticles form but silver sulfadiazine (AgSD) is the most efficient antibacterial agent especially for burn wound dressings.

Method: In this report, mechanical, structural, and antibacterial properties of PAN nanofibers incorporation with silver sulfadiazine are mainly focused. AgSD was loaded for the first time on electrospinning as well as self-synthesized AgSD on PAN nanofibers by solution immersion method and then compared the results of both.

Results: Occurrence of chemical reaction among the functional groups of AgSD and PAN were analyzed using FTIR, for both types of specimen. Morphological and surface properties of prepared nanofiber mats were characterized by scanning electron microscope, and it resulted in uniform nanofibers without bead formation. Diameter of nanofibers was slightly increased with addition of AgSD by in situ and immersion methods respectively. Nanoparticles distribution was analyzed by transmission electron microscopy. Thermal properties were analyzed by thermogravimetric analyzer and it was observed that AgSD decreased thermal stability of PAN which is better from biomedical perspective. X-ray diffraction declared crystalline structure of nanofiber mats. Presence of Ag and S contents in nanofiber mats was analyzed by X-ray photo spectroscopy. Antibacterial properties of nanofiber mats were investigated by disc diffusion method was carried out. E. coli and Bacillus bacteria strain were used as gram-negative and gram-positive respectively. Zone inhibition of the bacteria was used as a tool to determine effectiveness of AgSD released from PAN nanofiber mats. The antibacterial properties of PAN nanofibers impregnated with AgSD were determined with both types of bacteria strains to compare with control one.

Conclusion: On the basis of characterization results it is concluded that PAN/AgSD (immersion) nanofiber mats have better structural and antibacterial properties than that of PAN/AgSD (in situ) nanofiber mats. So, from our point of view, self-synthesized AgSD is recommended for further production of nanofiber mats for antibacterial applications.

Keywords: silver sulfadiazine, PAN nanofibers, antibacterial, solution immersion, in situ

\section{Introduction}

Advancement in technology has been beneficial for human beings, meanwhile it has imparted some negative impacts on some of the major areas, and health is one of the most affected areas among all of them. Biotechnology is also improving day by day by using advanced machinery as well as emerging materials which have better antibacterial, ${ }^{1-6}$ biodegradable, and biocompatible properties. Nanofiber mats have been used in biomedical applications since a few decades. Nanofibers are important 
part of biomedical technology, as $60 \%$ of the total nanofiber production is specifically used in biomedical applications, such as in tumor therapy, ${ }^{7}$ wound dressing, drug delivery, self-healing of wounds, or implants. ${ }^{8-11}$

Silver, incorporated with natural or synthetic polymers, has been used as an effective antibacterial agent since decades. Silver has potential applications in health care, especially in the form of nanoparticles. Silver sulfadiazine (AgSD) has been used for the treatment of second-order burns since early 1970s. ${ }^{12}$ Previously, it was considered that at lower concentrations, sulfadiazine group in AgSD was not effective in providing antibacterial activity but was effective either in combination with silver or at higher concentrations. ${ }^{13}$ Several studies were carried out to investigate the effect of cerium salt on inactivation of $\mathrm{AgSD}$, and it was observed that addition of cerium salts made the inhibition zone smaller in antibacterial assays, which was attributed to inactivation of AgSD.${ }^{14} \mathrm{At} \mathrm{first,}$ silver nanoparticales (AgNPs) were not accepted as a potent antimicrobial agent because of the availability of other good antibacterial agents in the market. The use of AgNPs as an antibacterial agent increased when electrospinning method was introduced because this technique resulted in an efficient use of metallic nanoparticles. ${ }^{15}$ Nylon/silver-based composite membranes were analyzed for structural applications, and it resulted in improved mechanical properties of composite membranes as compared to that of nylon membranes. ${ }^{16}$ Higher antibacterial activity was also observed in electrospun polyvinyl alcohol and chitosan incorporated with sliver nitrate $\left(\mathrm{AgNO}_{3}\right) .{ }^{17}$ Impregnation of AgNPs in nylon-6 by one-step direct electrospinning method has been characterized for surface, morphological, and antimicrobial activity as well. ${ }^{18}$

AgNPs have broad applications when incorporated with natural or synthetic polymers, ie, Zein, polymethyl methacrylate, chitosan, polyvinylpyrrolidone, polyacrylonitrile (PAN), and other polymers. ${ }^{11,19-21}$

PAN is a white, semi-crystalline organic synthetic polymer with chemical formula of $\mathrm{C}_{3} \mathrm{H}_{3} \mathrm{~N}$. PAN was synthesized in 1930 for the first time. It degrades above $300^{\circ} \mathrm{C} .{ }^{22} \mathrm{PAN}$ fibers having diameter $<1 \mu \mathrm{m}$ were successfully prepared in 1971, and the effect of flow rate of polymer on final properties was studied in detail. ${ }^{23}$ The effect of loading proportions of AgNPs (AgNPs were embedded by photocatalytic reduction of $\mathrm{AgNO}_{3}$ under UV irradiation) on $\mathrm{PAN} / \mathrm{TiO}_{2}$ was studied, and it was concluded that $\operatorname{AgNPs}(\sim 2 \mathrm{~nm}$ in size $)$ were greatly affected by the amount of $\mathrm{TiO}_{2}$ present in nanofibers. ${ }^{24}$ PAN-polyaniline (PANI) super-hydrophobic nanofiber mats were fabricated by in situ polymerization method which had water contact angle of $164.5^{\circ}$. The prepared nanofibers were also reversible to hydrophilic state within a short period of time. ${ }^{25}$ The PAN nanofiber membrane was successfully prepared and characterized for soybean oil hydrolysis process. It was done by immobilization of lipase on the membrane by covalent bonding. ${ }^{26}$ Similar technique was also used for biodiesel production from soybean oil. ${ }^{27} \mathrm{PAN}$ (core and shell) nanofiber mats incorporated with vitamin $\mathrm{C}$ and vitamin $\mathrm{E}$ extracts have been used in skin care applications, as they provide protection against damage by UV rays. ${ }^{28} \mathrm{PAN} / \mathrm{PVDF}$ nanofibers were surface modified by low vacuum plasma treatment for water remediation application. ${ }^{29} \mathrm{PAN}$ nanofibers are not only useful in medical applications but also cover other fields of science as well. Surface-modified PAN nanofibers have been also used as a substrate for identification of small molecules by surface-enhanced Raman scattering. ${ }^{30}$ Electrospinning is a useful technique to fabricate nanofibers having diameters ranging from tens of nanometer to microns. . $^{31,32}$ Electrospinning was introduced in 1931 and hence is not a new method for fabrication of nanofibers, but a proper setup for producing nanofibers by this method became practical and popular in the early 21 st century. From then onward, it has been used for the production of nanofibers for different applications, including water filtration, wound care, breathe masks, and other biomedical applications. Natural and synthetic fibers have been electrospun either in single form or blends of different types of polymers to obtain desired features. ${ }^{9,33-39}$ Figure 1 briefly illustrates the process of electrospinning.

Keeping in view the applications of PAN and AgSD in biomedical field, we prepared PAN/AgSD nanofiber mats for wound dressing. We are the first to load AgSD on electrospun nanofiber mats as well as perform fabrication of AgSD on nanofiber mats by immersion method. This research will be beneficial for other researchers as well as to industries in producing PAN nanofibers with better antimicrobial properties either by in situ loading of $\mathrm{AgSD}$ or deposition of $\mathrm{AgSD}$ on nanofiber mats by immersion method. Combination of mechanical and thermal properties of PAN nanofibers and antibacterial properties of AgSD would be effective for biomedical applications.

\section{Materials and methods Materials}

PAN polymer was purchased from Sigma-Aldrich Corporation (St Louis, MO, USA) in powder form and with an average molecular weight of 150,000 MW (typical). Sulfadiazine sodium salt (analytical standard, $\geq 98 \%$ ) was purchased from Sigma-Aldrich Corporation. $\mathrm{AgNO}_{3}$, with $99.8 \%$ purity, was purchased from Fujifilm Wako Pure Chemical Corporation (Osaka, Japan). N,N-Dimethylformamide (DMF) was purchased from Fujifilm Wako Pure Chemical Corporation. AgSD 


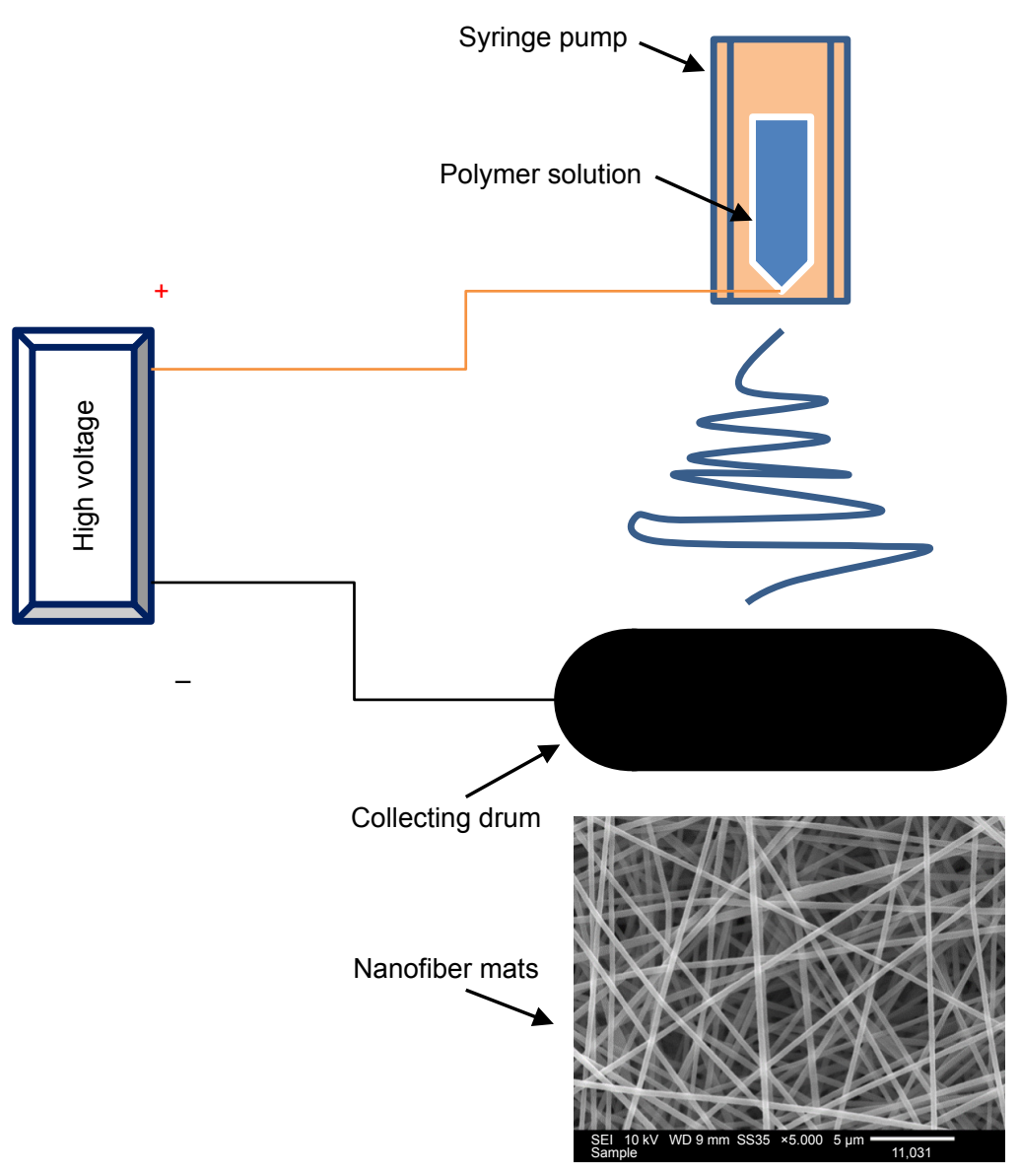

Figure I Schematic illustration of electrospinning process.

was purchased from Sigma-Aldrich Corporation in powder form with $98 \%$ purity. Sodium hydroxide $(\mathrm{NaOH})$ (in pellet form) was purchased from Fujifilm Wako Pure Chemical Corporation. Distilled water was used from the laboratory.

\section{Methods}

Samples were prepared by following three methods.

\section{Pure PAN nanofiber mats}

PAN ( $8 \%$ by weight) was dissolved in DMF, and the resulting solution was stirred continuously for 12 hours at room temperature $\left(25^{\circ} \mathrm{C} \pm 3^{\circ} \mathrm{C}\right)$. Then, the solution was loaded into the electrospinning apparatus for nanofiber production. Electrospinning conditions were as follows: syringe capacity $20 \mathrm{~mL}$, nozzle diameter $0.5 \mathrm{~mm}$, voltage was fixed at $15 \mathrm{kV}$, distance between nozzle and collector drum was kept at $14 \mathrm{~cm}$, and flow rate of solution was adjusted to $0.5 \mathrm{~mL} / \mathrm{h}$. Electrospinning conditions remained same for all the samples.

PAN/AgSD in situ electrospun (PAN/AgSD [ES]) For PAN/AgSD (ES) samples, firstly PAN ( $8 \%$ by weight) was dissolved in DMF for 12 hours and then $\mathrm{AgSD}(0.6 \%$ by weight $)$ was added to the PAN/DMF solution and kept on stirring for
2 hours. Then the solution was loaded onto the electrospinning apparatus with the conditions similar to that of sample 1.

\section{Self-synthesized AgSD on PAN nanofibers} (PAN/AgSD immersion)

For PAN/AgSD immersion samples, in the first step, pure PAN nanofiber mats were electrospun as per the conditions described for sample 1, and then samples were placed in glass trays which contained aqueous solution of sulfadiazine sodium salt $(0.2 \%$ by weight $)$ and $\mathrm{AgNO}_{3}(0.1 \%$ by weight $)$. The trays were placed in a controlled stirring chamber for 24 hours and then the samples were removed from solution of sulfadiazine and $\mathrm{AgNO}_{3}$ and were placed in $0.1 \%$ by weight aqueous solution of $\mathrm{NaOH}$ for 4 hours to enable reduction of $\mathrm{AgNO}_{3}$. Samples were then dried in a vacuum oven at $30^{\circ} \mathrm{C}$ for 12 hours. Samples were kept in air-tight bags for further testing.

\section{Characterizations}

Antibacterial properties were determined by disc diffusion method where strains of Escherichia coli (BUU25113) and Bacillus (B-sub 168) bacteria were used as representatives of Gram-negative and Gram-positive bacteria, respectively. The nanofiber mats were cut into discs having $8 \mathrm{~mm}$ diameter 
and were placed on top of two different plates, which were cultured with each bacterial strain and incubated overnight for 12 hours at $37^{\circ} \mathrm{C}$. The morphological properties of the prepared nanofiber mats were determined by using scanning electron microscope (SEM, JSM-5300; JEOL Ltd, Tokyo, Japan), which was accelerated with the voltage of $10 \mathrm{kV}$, and transmission electron microscope (JEM-2100; JEOL Ltd), accelerated with $200 \mathrm{kV}$. An image analysis software (ImageJ, version 1.4.3) was used to calculate the average diameters of nanofibers by taking 50 readings randomly for each sample. Wide-angle $\mathrm{X}$-ray diffraction was performed for the determination of the crystal structure (at $25^{\circ} \mathrm{C}$ ) of nanofiber mats using nickel-filtered $\mathrm{Cu}$ Ka radiation-assisted Rotaflex RT300 mA (Rigaku Corporation, Osaka, Japan), with an angular angle of $5 \leq 2 \theta \leq 80^{\circ}$. The chemical reactivity among PAN and AgSD functional groups was characterized by using Fourier transform infrared spectroscopy (FTIR) with an attenuated total reflectance (ATR) probe Prestige-21 (Shimadzu, Tokyo, Japan), and wavelength for ATR spectra was set in the range of $400 \mathrm{~cm}^{-1}$ to $4,000 \mathrm{~cm}^{-1}$. Thermal degradation of PAN, PAN/AgSD (ES), and PAN/AgSD (immersion) was studied by thermogravimetric analysis (TGA) using thermo-plus TG 8120 (Rigaku Corporation) which was run under ambient conditions (room temperature) at a heating rate of $10^{\circ} \mathrm{C} / \mathrm{min}$ and temperature range was set at $0^{\circ} \mathrm{C}-500^{\circ} \mathrm{C}$ for all samples. The X-ray photo-spectroscopy (XPS) analysis was performed by using Shimadzu-Kratos AXIS-ULTRA HAS SV (Shimadzu). Mechanical properties (tensile strength, strain, and modulus) of PAN and PAN/AgSD nanofibers were determined by using the Universal Testing Machine (Tesilon RTC 250A; A\&D Company Ltd., Tokyo, Japan). Five specimens for each sample were prepared, according to
ISO 13634. Test was run at room temperature and crosshead speed was kept as $5 \mathrm{~mm} / \mathrm{min}$. From universal testing machine (UTM) data, values of strain, stress, and Young's modulus were calculated by equations 1,2 , and 3 , respectively,

$$
\begin{gathered}
\varepsilon=\frac{\Delta \mathrm{l}}{\mathrm{l}} \\
\sigma=\frac{\mathrm{F}}{\mathrm{A}} \\
\mathrm{E}=\frac{\sigma}{\varepsilon}
\end{gathered}
$$

where $\varepsilon, \sigma$, and $\mathrm{E}$ are strain, stress, and Young's modulus; $\Delta \mathrm{l}$ is change in length; 1 is original length of specimen; $F$ is applied force; and $\mathrm{A}$ is cross-sectional area of specimen.

\section{Antibacterial activity test}

Disc diffusion method was opted to investigate antibacterial properties of prepared nanofiber mats as shown in Figure 2A (E. coli) and B (Bacillus). E. coli and Bacillus strains were subjected to zone inhibition test. The diameter of zone inhibition around the disc was determined to evaluate the effectiveness of antibacterial property of the nanofibers (due to AgSD released from PAN nanofibers). The antibacterial activity of AgSD-loaded PAN nanofiber mats was taken into account for both bacterial strains and compared with control sample. Excellent antibacterial efficiency was observed for both samples, while sample having AgSD by immersion method (PAN/AgSD [immersion]) showed consistent results for both types of bacteria. It was detected that as the amount of AgSD was increased in samples, it had a significant

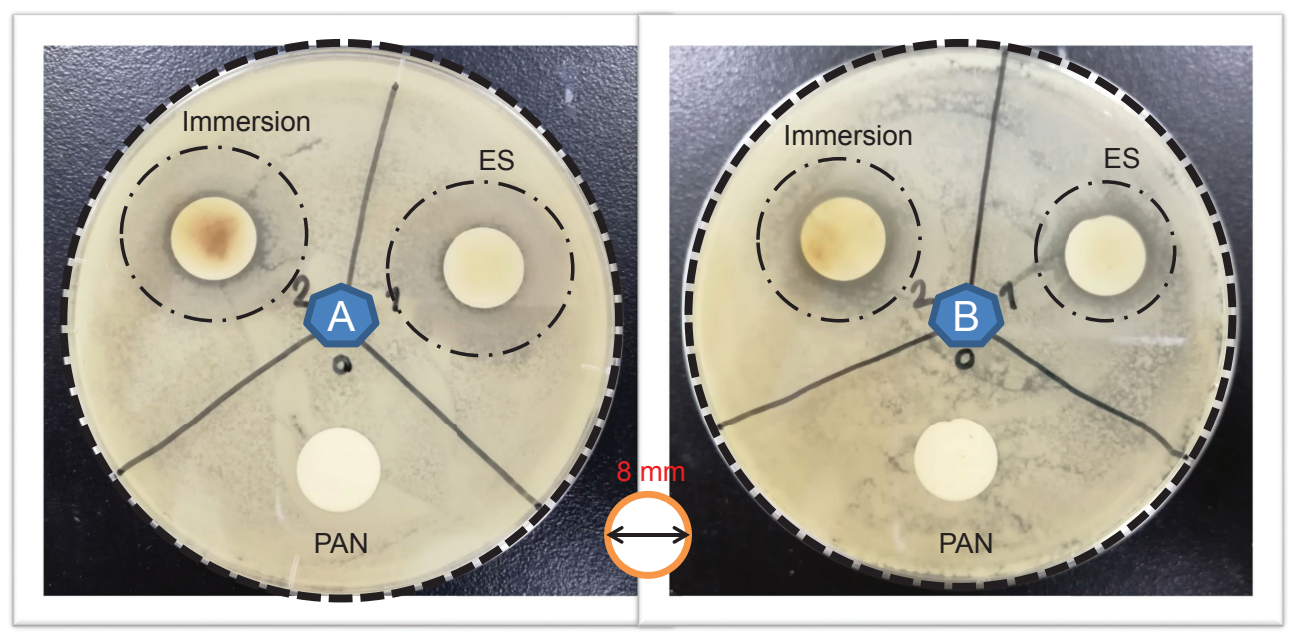

Figure 2 Antibacterial activity test: (A) Escherichia coli and (B) Bacillus.

Abbreviations: PAN, polyacrylonitrile; ES, in situ electrospun. 
influence on the antibacterial property of nanofiber mats. Herein, from the results it was concluded that self-synthesized AgSD on PAN nanofibers showed better antibacterial activity as compared to in situ added AgSD in PAN nanofibers.

\section{Morphological properties}

Morphological properties of prepared nanofiber mats were determined by SEM. The SEM images in Figure 3 show that nanofibers of pure PAN were smooth without any bead formation under specified conditions. Addition of AgSD by in situ method affected the smoothness of electrospun fibers, as they became slightly rough and a slight increment in diameter was also noted. SEM image of samples in which AgSD was incorporated by immersion method showed that AgSD got entangled in between PAN nanofibers and AgSD nanocrystals were also observed on the surface of nanofibers. Diameter of PAN/AgSD (immersion) samples was also slightly greater than both of the previous samples.

Figure 4 shows nanoparticle distribution on the nanofibers. It can be observed that AgSD nanoparticles were evenly distributed on the surface of nanofibers which were prepared by direct in situ method, while AgSD synthesized by immersion method showed nanoparticles in the form of grafts. The surface of nanofibers was also covered by AgSD grafts.

\section{Nanofiber diameter}

As we mentioned earlier, diameter of the nanofibers was altered by the impregnation of AgSD into the PAN either by in situ method or immersion method. Figure 5 shows quantitative explanation of average diameters of nanofibers. The average of 50 nanofibers from each sample exhibited slight variation in diameters. In Figure 5, it can be noticed that average diameters of PAN, PAN/AgSD (ES), and PAN/ $\mathrm{AgSD}$ (immersion) were 141.94, 145.02, and $146.94 \mathrm{~nm}$, respectively. Although there was a slight increment in diameters with addition of AgSD in PAN nanofibers, these diameters were within the range of SD of all the samples. So, we can conclude that there was a slight or no effect of $\mathrm{AgSD}$ on the diameter of PAN nanofibers.

\section{EDX analysis}

Energy-dispersive X-ray (EDX) analysis was carried out for the quantitative assessment of sulfur and Ag contents in the nanofibers. It can be observed in Figure 6 that sample A has
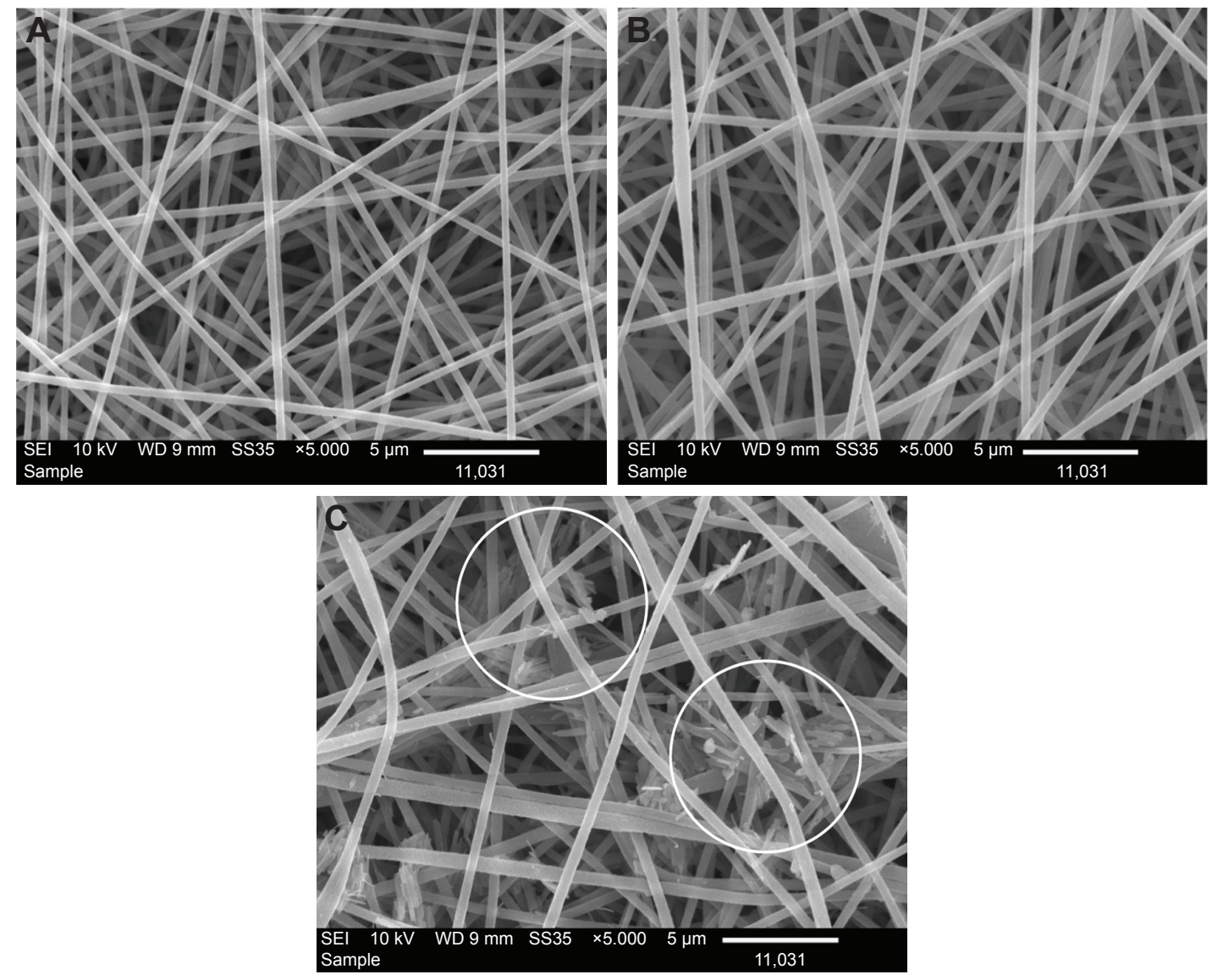

Figure 3 SEM images of (A) PAN nanofibers, (B) PAN/AgSD (ES) nanofibers, and (C) PAN/AgSD (immersion) nanofibers.

Abbreviations: SEM, scanning electron microscope; PAN, polyacrylonitrile; AgSD, silver sulfadiazine; ES, in situ electrospun. 

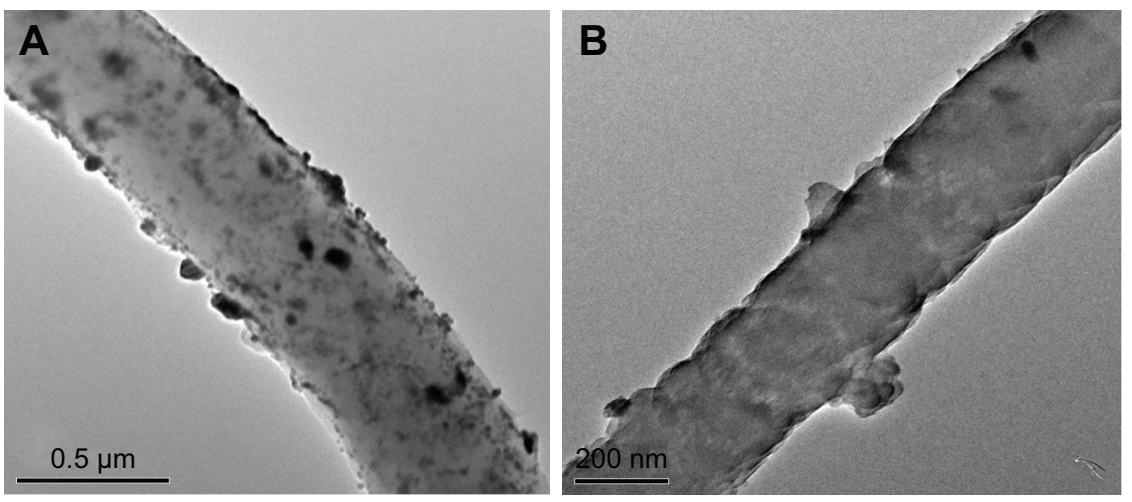

Figure 4 TEM images of (A) PAN/AgSD (ES) nanofiber mats and (B) PAN/AgSD (immersion) nanofiber mats.

Abbreviations: TEM, transmission electron microscope; PAN, polyacrylonitrile; AgSD, silver sulfadiazine; ES, in situ electrospun.

lower values of $\mathrm{Ag}$ and $\mathrm{S}$ as compared to that of sample $\mathrm{B}$. It was concluded that samples having self-synthesized AgSD by immersion method had higher values of both $\mathrm{Ag}$ as well as S. Furthermore, it was also proven by antibacterial activity test that self-synthesized AgSD on PAN nanofibers show excellent results.

\section{FTIR interpretation}

FTIR spectra of pure PAN and composite nanofibers of PAN/ AgSD are shown in Figure 7. It was observed that AgSD had shown sharp peaks at 1,203 and 1,231.6 $\mathrm{cm}^{-1}$, which can be associated with the main functionality of $\mathrm{AgSD}$, that is, $\mathrm{SO}_{2}$ asymmetric stretching. AgSD-loaded nanofibers had also represented peaks at $1,561 \mathrm{~cm}^{-1}, 1,583 \mathrm{~cm}^{-1}$, and $3,410 \mathrm{~cm}^{-1}$, which can be associated with stretching band of amine $\left(\mathrm{NH}_{2}\right)$ group. It was interesting to observe that the spectra of pure PAN nanofibers were not affected by impregnation of AgSD in PAN nanofibers, which indicates that there may be no

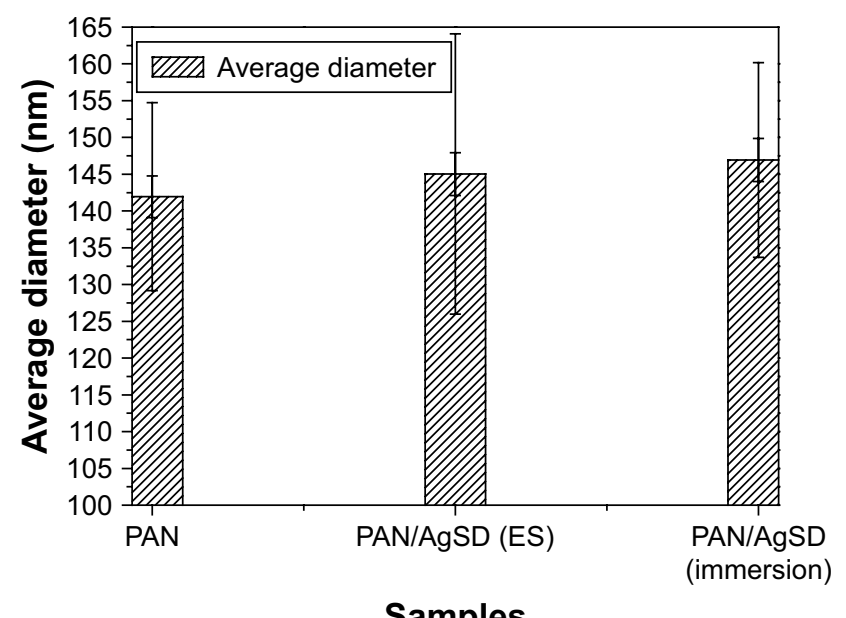

Figure 5 Diameter interpretation of PAN and PAN/AgSD nanofibers. Abbreviations: $\mathrm{PAN}$, polyacrylonitrile; $\mathrm{AgSD}$, silver sulfadiazine; $\mathrm{ES}$, in situ electrospun. chemical reaction between PAN and AgSD. ${ }^{29,30}$ PAN showed prominent peaks at $1,452 \mathrm{~cm}^{-1}, 1,664 \mathrm{~cm}^{-1}, 2,240 \mathrm{~cm}^{-1}$, and $2,923 \mathrm{~cm}^{-1}$. Peaks at $1,664 \mathrm{~cm}^{-1}, 2,240 \mathrm{~cm}^{-1}$, and 2,923 $\mathrm{cm}^{-1}$ represent stretching vibrations of $-\mathrm{C}=\mathrm{N},-\mathrm{C} \equiv \mathrm{N}$, and $-\mathrm{CH}$ groups, respectively, while the peak at $1,452 \mathrm{~cm}^{-1}$ represents bending vibration of $-\mathrm{CH} 2$ group. It was observed that the characteristic peak of PAN nanofibers at wavelength of $1,664 \mathrm{~cm}^{-1}$ slightly decreased with in situ addition of AgSD in PAN, while the same peak became shorter with the addition of AgSD by immersion method. It was also observed that PAN/AgSD (immersion) samples showed higher intensity and sharper peaks than PAN/AgSD (ES) samples.

\section{X-ray diffraction (XRD)}

XRD was studied to check crystalline structure of nanofiber mats. Pure PAN nanofibers (Figure 8) showed a sharp peak at $2 \theta=17^{\circ}$ and a small peak at $2 \theta=30^{\circ}$, while PAN did not exhibit any peak up to $2 \theta=80^{\circ}$. PAN nanofibers showed characteristic peaks of pure PAN. First peak $\left(17^{\circ}\right)$ could be associated with hexagonal lattice of PAN. Impregnation of AgSD in PAN exhibited sharp peaks at $2 \theta=9^{\circ}, 2 \theta=11^{\circ}$, $2 \theta=21^{\circ}$, and many shorter peaks from $2 \theta=30^{\circ}$ to $2 \theta=60^{\circ}$. All other peaks, except for the two peaks at $2 \theta=17^{\circ}$ and $2 \theta=30^{\circ}$, represented the presence of AgSD and all those were linked with AgSD. XRD plot also revealed that stability of PAN was altered by the impregnation of $\mathrm{AgSD}$, as intensity of the characteristic peak $\left(2 \theta=17^{\circ}\right)$ decreased with the addition of AgSD. It was observed that PAN/AgSD (immersion) sample showed higher intensity peaks than those of PAN/AgSD (ES) samples. The presence of AgSD in PAN nanofibers was also confirmed by XPS analysis.

\section{Thermogravimetric analysis}

TGA was performed to analyze the significance of AgSD on thermal properties of PAN. Also, the effect of in situ added 

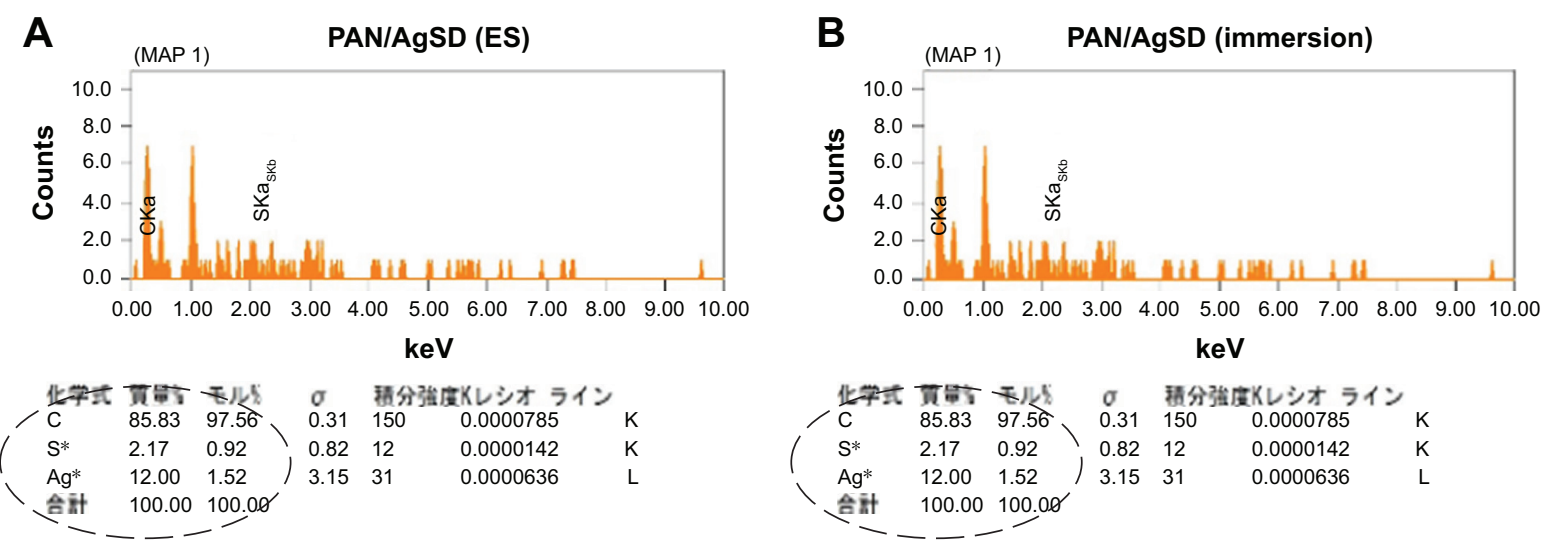

Figure 6 EDX analysis of PAN/AgSD (ES) (A) and PAN/AgSD (immersion) (B) nanofibers.

Notes: Dotted circles shown presence of $\mathrm{Ag}, \mathrm{C} \& \mathrm{~S}$. *Under observation.

Abbreviations: EDX, energy-dispersive X-ray; PAN, polyacrylonitrile; AgSD, silver sulfadiazine; ES, in situ electrospun.

and self-synthesized AgSD on thermal properties of PAN nanofibers was taken under consideration. TGA curve is generally separated into three parts: first part (up to $100^{\circ} \mathrm{C}$ ) represents moisture removal, second part of the curve indicates thermal degradation onset and offset as well, and the last part indicates combustion zone where residual amount is measured. It can be observed in Figure 9 that the first part of TGA curve indicates that higher amount of moisture is absorbed by PAN/AgSD (immersion) nanofibers than PAN nanofibers and PAN/AgSD (ES) nanofiber mats. The second part of TGA curve, which indicates the onset temperature, showed that the onset of pure PAN nanofibers was at $320^{\circ} \mathrm{C}$. PAN/AgSD (ES) showed onset at $290^{\circ} \mathrm{C}$ and onset of PAN/ AgSD (immersion) occurred at $190^{\circ} \mathrm{C}$. PAN/AgSD (ES) nanofibers showed a sharp onset, while PAN/AgSD (immersion) showed a gradual onset. Residual amounts of PAN

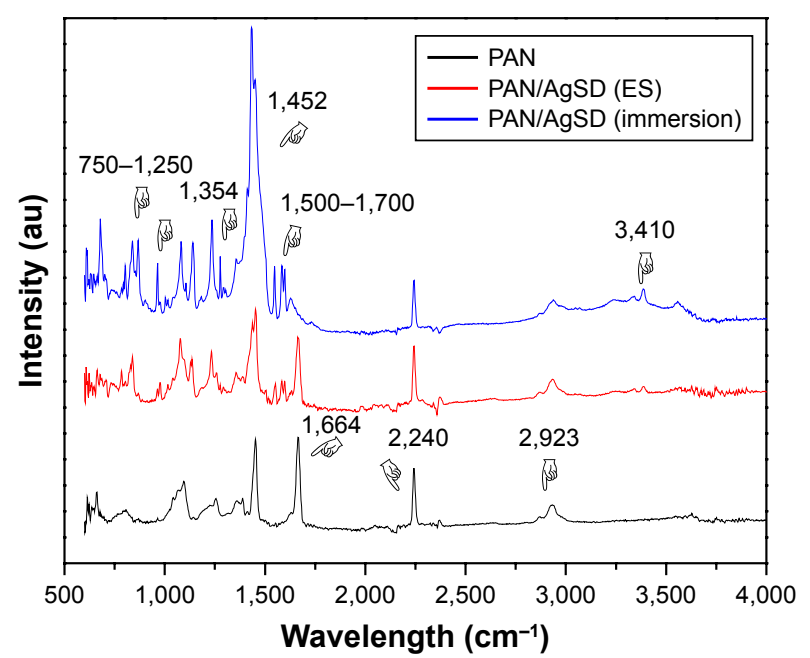

Figure 7 FTIR-ATR spectra of PAN and PAN/AgSD nanofiber mats. Abbreviations: FTIR, Fourier transform infrared spectroscopy; ATR, attenuated total reflectance; PAN, polyacrylonitrile; AgSD, silver sulfadiazine; ES, in situ electrospun. nanofibers, PAN/AgSD (ES), and PAN/AgSD (immersion) were $58 \%, 44 \%$, and $32 \%$, respectively. From TGA data, it was decided that impregnation of AgSD resulted in low thermal stability to PAN. The excellent thermal stability of PAN was decreased by the addition of AgSD, making it readily degradable, which is considered as a good characteristic for biomedical applications.

\section{X-ray spectroscopy}

XPS was performed to confirm the existence of AgSD in nanofiber mats (Figure 10). As AgSD is composed of $\mathrm{Ag}$ (silver) and S (sulfur), XPS spectra for both Ag ( $3 \mathrm{~d}$ and $3 p$ ) and sulfur ( $2 \mathrm{~s}$ and $2 \mathrm{p}$ ) were obtained. Ag $3 \mathrm{p}$ spectra showed two peaks at $574.0 \mathrm{eV}\left(\mathrm{Ag} 3 \mathrm{p}_{3 / 2}\right)$ and $606.0 \mathrm{eV}\left(\mathrm{Ag} 3 \mathrm{p}_{1 / 2}\right)$ with a split of $32 \mathrm{eV}$. Spectra of $\mathrm{Ag} 3 \mathrm{~d}$ showed peaks at $370.0 \mathrm{eV}$ and $376.5 \mathrm{eV}$ with a split of $6.5 \mathrm{eV}$. These peaks can be

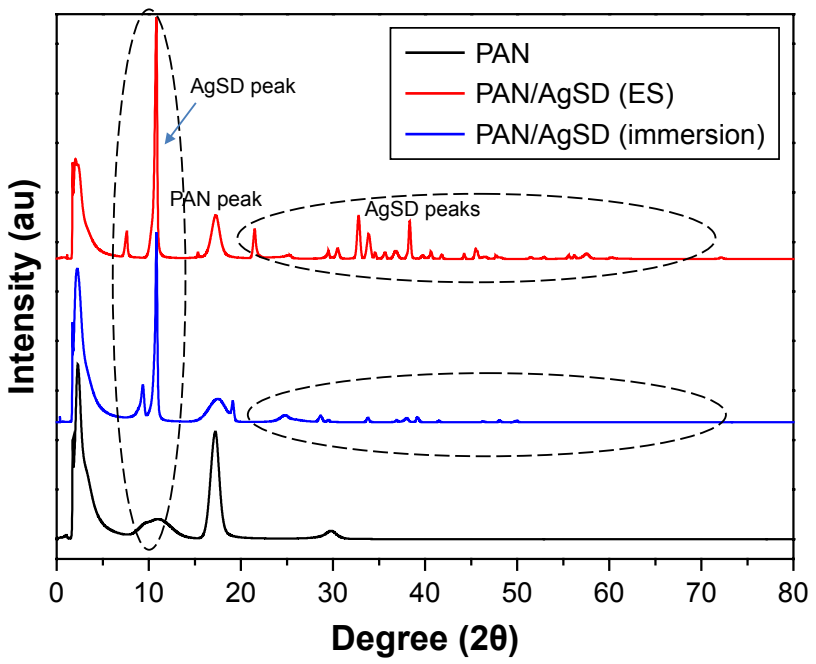

Figure $8 \times R D$ analysis of PAN nanofibers and PAN/AgSD nanofibers. Abbreviations: $X R D, X$-ray diffraction; $P A N$, polyacrylonitrile; $A g S D$, silver sulfadiazine; $\mathrm{ES}$, in situ electrospun. 


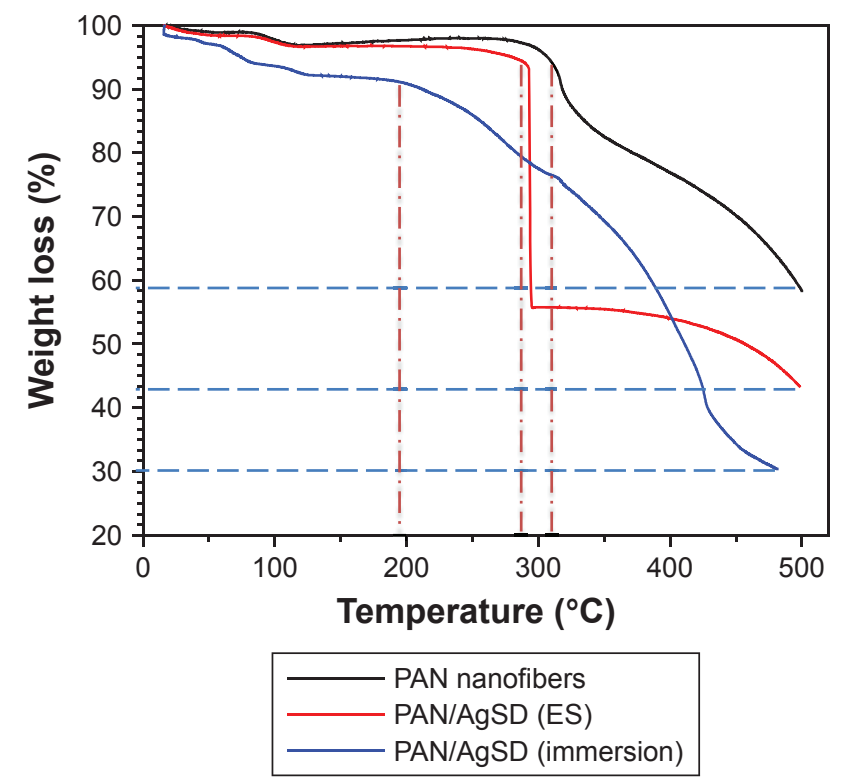

Figure 9 Thermal degradation analysis of PAN nanofibers and PAN/AgSD nanofibers. Abbreviations: PAN, polyacrylonitrile; $\mathrm{AgSD}$, silver sulfadiazine; $\mathrm{ES}$, in situ electrospun.

A

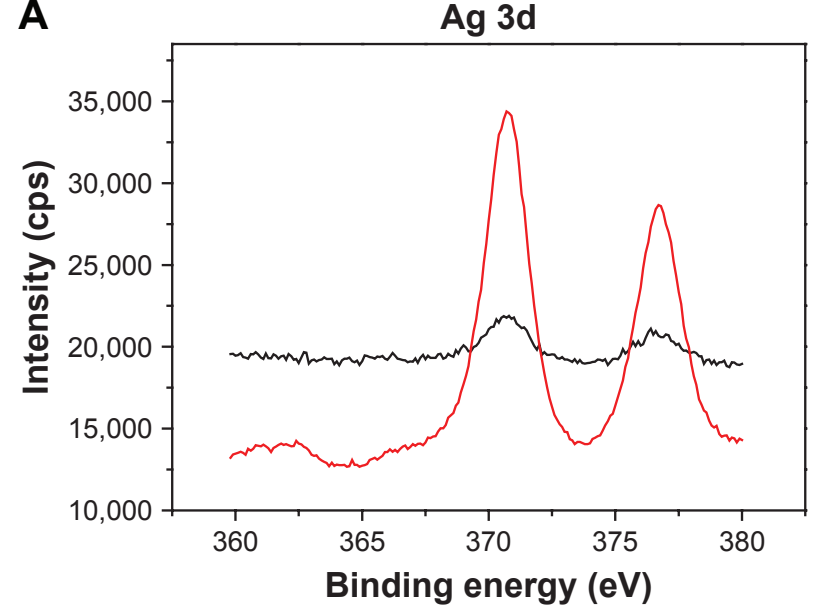

C

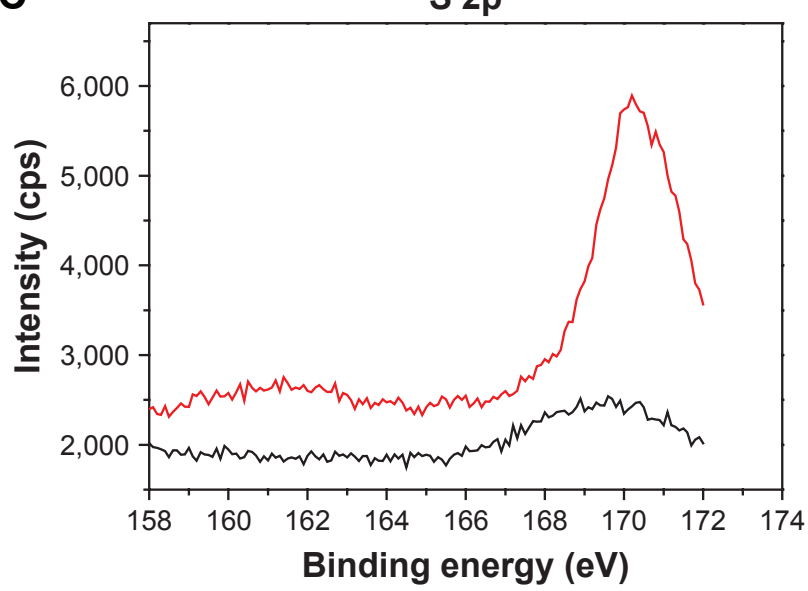

correlated to metallic $\mathrm{Ag}$ present in nanofibers. The standard peaks for $\mathrm{Ag} 3 \mathrm{p}_{3 / 2}$ and $\mathrm{Ag} 3 \mathrm{p}_{1 / 2}$ were noted at $570.0 \mathrm{eV}$ and $604.0 \mathrm{eV}$, respectively, while standard peaks for $\mathrm{Ag} 3 \mathrm{~d}_{5 / 2}$ and $\mathrm{Ag} 3 \mathrm{~d}_{3 / 2}$ were noted at $368 \mathrm{eV}$ and $374.5 \mathrm{eV}$, respectively. Spectra of S 2s showed peak at $234.0 \mathrm{eV}$ and that of S $2 p$ at $169.0 \mathrm{eV}$. However, in case of PAN/AgSD (ES) sample, it was observed that $\mathrm{S} 2 \mathrm{~s}$ showed one more peak at $239 \mathrm{eV}$, while standard peaks for S $2 \mathrm{~s}$ and $\mathrm{S} 2 \mathrm{p}$ were noted at $231.0 \mathrm{eV}$ and $164.5 \mathrm{eV}$, respectively. It can be seen in Figure 10 that all relevant peaks for $\mathrm{Ag}$ and $\mathrm{S}$ are sharper and have higher intensity for PAN/AgSD (immersion) samples as compared to that of PAN/AgSD (ES) samples. XPS spectra provided clear evidence for the presence of silver and sulfur in the nanofiber mats.

\section{Mechanical properties}

The mechanical properties (tensile strength, strain, Young's modulus) of the prepared samples were analyzed. Table 1
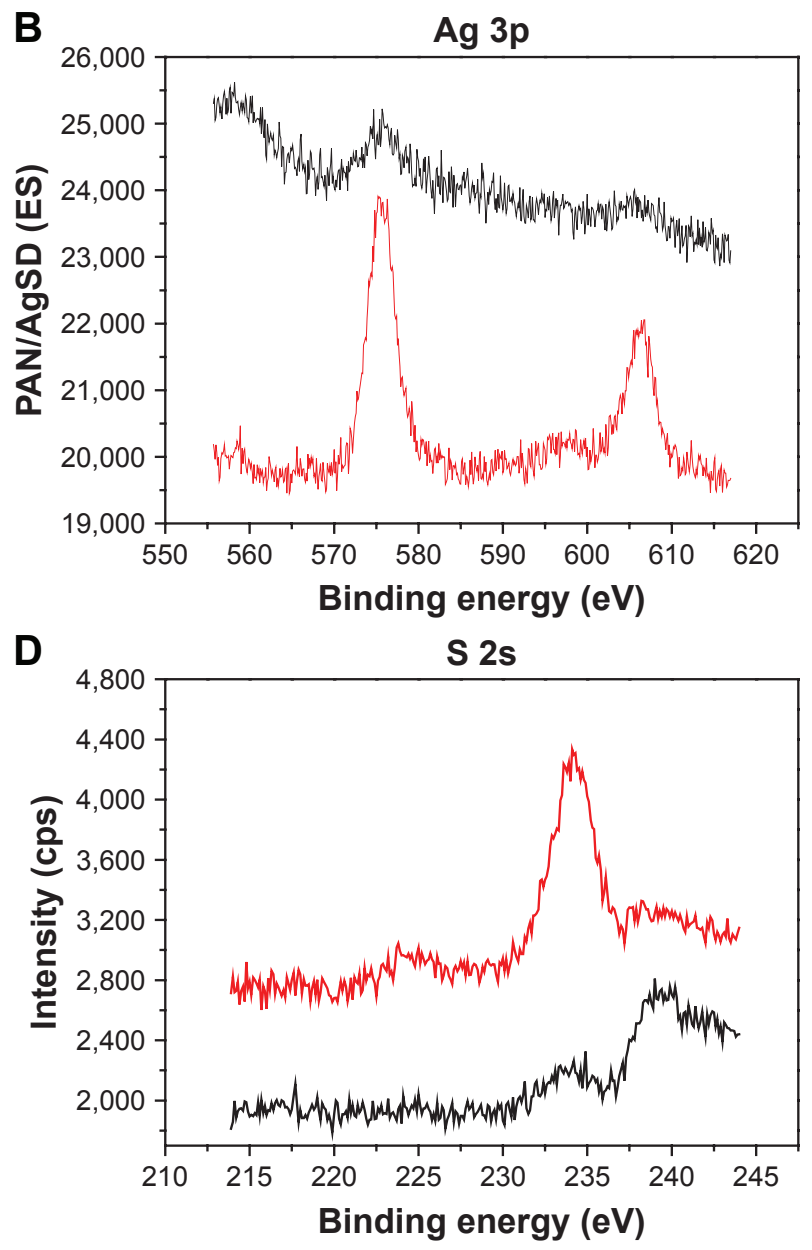

PAN/AgSD (ES) — PAN/AgSD (immersion)

Figure 10 XPS analysis of PAN/AgSD (ES) and PAN/AgSD (immersion): (A) Ag 3d, (B) Ag 3p, (C) S 2p, and (D) S $2 s$.

Abbreviations: XPS, X-ray photo-spectroscopy; PAN, polyacrylonitrile; AgSD, silver sulfadiazine; ES, in situ electrospun. 
Table I Summary of mechanical properties of PAN nanofibers and PAN/AgSD nanofibers

\begin{tabular}{l|l|l|l}
\hline Properties & PAN nanofibers & PAN/AgSD (ES) & PAN/AgSD (immersion) \\
\hline Tensile Strength (MPa) & $3.984 \pm 0.186$ & $1.728 \pm 0.263$ & $4.163 \pm 0.314$ \\
Elongation at break (\%) & $11.254 \pm 2.317$ & $14.087 \pm 1.971$ & $41.004 \pm 4.035$ \\
Young's modulus (MPa) & 35.400 & 12.613 & 10.153 \\
\hline
\end{tabular}

Note: Data are presented as mean \pm SD.

Abbreviations: PAN, polyacrylonitrile; AgSD, silver sulfadiazine; $E S$, in situ electrospun.

sums up all results obtained from UTM data. It can also be shown in Figure 11 that PAN nanofibers showed higher tensile strength (3.984 MPa), while in situ addition of AgSD decreased the tensile strength of PAN. It can be related with weak physical attraction between AgSD and PAN. The results of FTIR spectra revealed that there was no chemical reaction between AgSD and PAN as the peaks of PAN were retained in the blended form too. It was interesting to observe that the tensile strength of PAN/AgSD (immersion) sample was increased (4.163 MPa), which is an indication of stronger physical bonding between AgSD and PAN nanofibers. In SEM images (Figure 3), it was observed that PAN/AgSD (immersion) nanofibers have entangled AgSD in between the joints of nanofibers as well as on the surface of nanofibers, which gives mechanical stability to PAN nanofibers.

With regard to elongation at break, it can be viewed in Figure 11 that elongation at break increased with addition of AgSD, either by in situ method or by immersion method. However, PAN/AgSD (immersion) nanofiber mats showed higher value of elongation at break than that of PAN/AgSD (ES) nanofiber mats. Figure 12 shows bar chart of tensile strength of all samples with SD. Young's modulus is also one of the important performance parameters.

Figure 13 shows pictorial analysis and Table 1 shows quantitative data of Young's modulus of the samples. It was

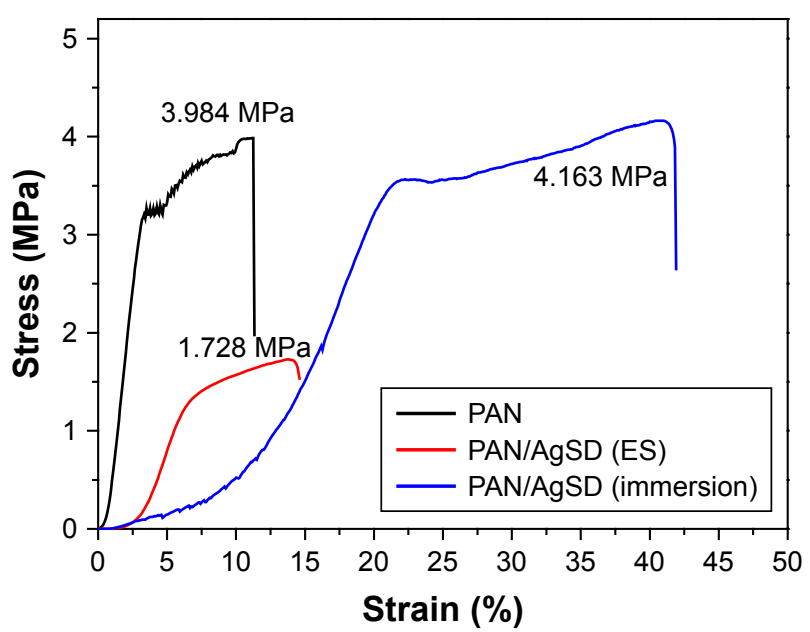

Figure I I Stress-strain curve of PAN nanofibers and PAN/AgSD nanofibers. Abbreviations: $\mathrm{PAN}$, polyacrylonitrile; $\mathrm{AgSD}$, silver sulfadiazine; $\mathrm{ES}$, in situ electrospun. observed that the value of Young's modulus gradually decreased with the addition of AgSD by in situ and immersion methods.

\section{Conclusion}

Keeping in mind all the results and discussions of the prepared specimens, it can be concluded that PAN retains its structure and basic characteristics with the addition of AgSD. Antibacterial activity test showed excellent antibacterial results for both PAN/AgSD (ES) and PAN/AgSD (immersion) nanofiber mats. From SEM results, it is clear that nanofiber structure was not much disturbed by $\mathrm{AgSD}$. The surface of nanofiber became slightly rough, which is considered to be helpful for antibacterial activity. FTIR spectra showed characteristic peaks of AgSD and PAN in nanofibers. No shift in the peak was observed, hence it can be inferred that there was no physical bonding between PAN and AgSD. XPS spectra clearly showed the presence of Ag and $\mathrm{S}$ in nanofiber mats. It was observed that for all the above-mentioned characterizations, PAN/AgSD (immersion) nanofibers showed prominent results. XRD analysis showed that PAN has crystalline structure which was confirmed by the peak at $2 \theta=17^{\circ}$, which was associated with hexagonal

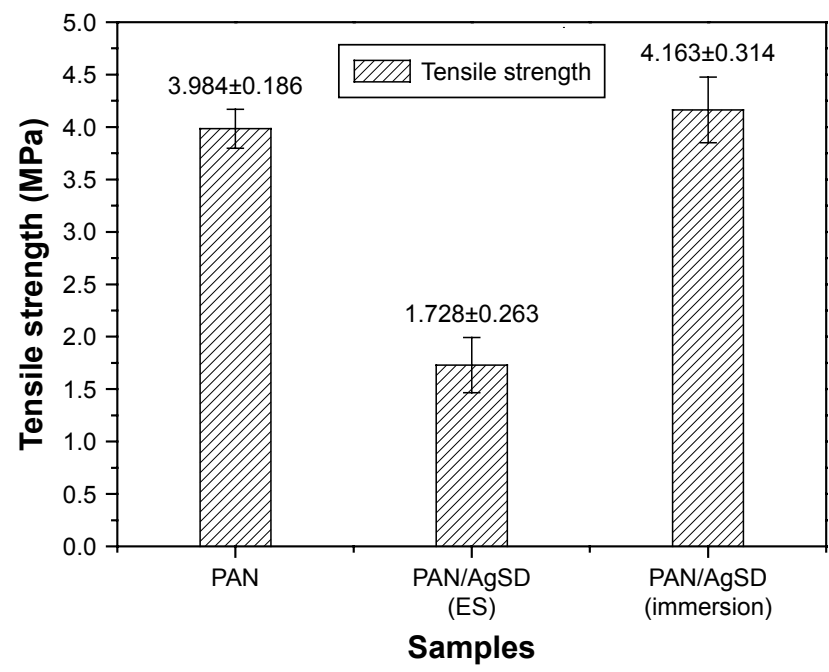

Figure 12 Comparison of tensile strength of PAN nanofibers, PAN/AgSD (ES), and PAN/AgSD (immersion) nanofibers.

Abbreviations: PAN, polyacrylonitrile; AgSD, silver sulfadiazine; $\mathrm{ES}$, in situ electrospun. 


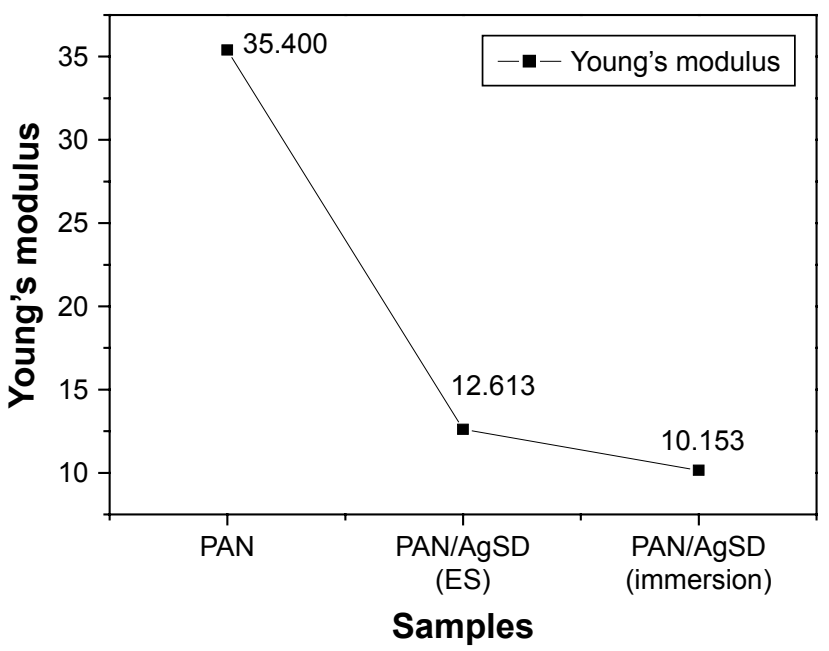

Figure 13 Comparison of Young's modulus of PAN nanofibers, PAN/AgSD (ES), and PAN/AgSD (immersion).

Abbreviations: PAN, polyacrylonitrile; AgSD, silver sulfadiazine; $E S$, in situ electrospun.

lattice of PAN, was decreased with addition of AgSD. It was further observed in the TGA study that thermal degradation of PAN/AgSD (ES) and PAN/AgSD (immersion) was lower than PAN nanofibers. Tensile strength and elongation at break was increased by addition of AgSD by immersion technique, while tensile strength was decreased with in situ addition of AgSD in PAN nanofibers during electrospinning. Taken together, it can be concluded that PAN/AgSD (immersion) nanofiber mats have better structural and antibacterial properties than PAN/AgSD (ES) nanofiber mats. So, from our point of view, self-synthesized AgSD is recommended for further production of nanofiber mats for antibacterial applications.

\section{Disclosure}

The authors report no conflicts of interest in this work.

\section{References}

1. Lin L, Dai Y, Cui H. Antibacterial poly(ethylene oxide) electrospun nanofibers containing cinnamon essential oil/beta-cyclodextrin proteoliposomes. Carbohydr Polym. 2017;178(June):131-140.

2. Cui H, Bai M, Lin L. Plasma-treated poly(ethylene oxide) nanofibers containing tea tree oil/beta-cyclodextrin inclusion complex for antibacterial packaging. Carbohydr Polym. 2018;179:360-369.

3. Lin L, Zhu Y, Li C, Liu L, Surendhiran D, Cui H. Antibacterial activity of PEO nanofibers incorporating polysaccharide from dandelion and its derivative. Carbohydr Polym. 2018;198(February):225-232.

4. Cui H, Bai M, Rashed MMA, Lin L. The antibacterial activity of clove oil/chitosan nanoparticles embedded gelatin nanofibers against Escherichia coli O157:H7 biofilms on cucumber. Int J Food Microbiol. 2018;266:69-78.

5. Lin L, Mao X, Sun Y, Rajivgandhi G, Cui H. Antibacterial properties of nanofibers containing chrysanthemum essential oil and their application as beef packaging. Int J Food Microbiol. 2019;292:21-30.

6. Cui H, Wu J, Li C, Lin L. Improving anti-Listeria activity of cheese packaging via nanofiber containing nisin-loaded nanoparticles. LWT - Food Science and Technology. 2017;81:233-242.
7. Wu C, Zhao J, Hu F, et al. Design of injectable agar-based composite hydrogel for multi-mode tumor therapy. Carbohydr Polym. 2018;180: $112-121$.

8. Kharaghani D, Kee Jo Y, Khan MQ, Jeong Y, Cha HJ, Kim IS. Electrospun antibacterial polyacrylonitrile nanofiber membranes functionalized with silver nanoparticles by a facile wetting method. Eur Polymer $J$. 2018;108(August):69-75.

9. Khan MQ, Kharaghani D, Nishat N, et al. The development of nanofiber tubes based on nanocomposites of polyvinylpyrrolidone incorporated gold nanoparticles as scaffolds for neuroscience application in axons. Text Res J. 2018. DOI:10.1177/0040517518801185

10. Lee H, Xu G, Kharaghani D, et al. Electrospun tri-layered zein/PVP$\mathrm{GO} /$ zein nanofiber mats for providing biphasic drug release profiles. Int J Pharm. 2017;531(1):101-107.

11. Dashdorj U, Reyes MK, Unnithan AR, et al. Fabrication and characterization of electrospun zein/Ag nanocomposite mats for wound dressing applications. Int J Biol Macromol. 2015;80:1-7.

12. Ullah S, Hashmi M, Khan MQ, et al. Silver sulfadiazine loaded zein nanofiber mats as a novel wound dressing. RSC Adv. 2019;9(1):268-277.

13. Fox CL, Modak SM. Mechanism of silver sulfadiazine action on burn wound infections. Antimicrob Agents Chemother. 1974;5(6):582-588.

14. Holder AI. In vitro inactivation of silver sulphadiazine by the addition of cerium salts. Burns. 1982;8(4):274-277.

15. Rai M, Yadav A, Gade A. Silver nanoparticles as a new generation of antimicrobials. Biotechnol Adv. 2009;27(1):76-83.

16. Francis L, Giunco F, Balakrishnan A, Marsano E, Synthesis ME. Synthesis, characterization and mechanical properties of nylon-silver composite nanofibers prepared by electrospinning. Curr Appl Phys. 2010; 10(4):1005-1008.

17. Hang AT, Tae B, Park JS. Non-woven mats of poly(vinyl alcohol)/ chitosan blends containing silver nanoparticles: Fabrication and characterization. Carbohydr Polym. 2010;82(2):472-479.

18. Pant B, Pant HR, Pandeya DR, et al. Characterization and antibacterial properties of Ag NPS loaded nylon-6 nanocomposite prepared by onestep electrospinning process. Colloids Surf A. 2012;395:94-99.

19. Ghavaminejad A, Rajan Unnithan A, Ramachandra Kurup SA, et al. Mussel-inspired electrospun nanofibers functionalized with sizecontrolled silver nanoparticles for wound dressing application. ACS Appl Mater Interfaces. 2015;7(22):12176-12183.

20. Yang C-H, Wang L-S, Chen S-Y, et al. Microfluidic assisted synthesis of silver nanoparticle-chitosan composite microparticles for antibacterial applications. Int J Pharm. 2016;510(2):493-500.

21. Maharjan B, Joshi MK, Tiwari AP, Park CH, Kim CS. In-situ synthesis of AgNPs in the natural/synthetic hybrid nanofibrous scaffolds: fabrication, characterization and antimicrobial activities. JMech Behav Biomed Mater. 2017;65:66-76.

22. Gupta AK, Paliwal DK, Bajaj P. Melting behavior of acrylonitrile polymers. J Appl Polym Sci. 1998;70(13):2703-2709.

23. Subbiah T, Bhat GS, Tock RW, Parameswaran S, Ramkumar SS. Nanofibers Eof. Electrospinning of nanofibers. J Appl Polym Sci. 2005;96(2):557-569.

24. Lim SK, Lee S-K, Hwang S-H, Kim H. Photocatalytic deposition of silver nanoparticles onto organic/inorganic composite nanofibers. Macromol Mater Eng. 2006;291(10):1265-1270.

25. Zhu Y, Feng L, Xia F, Zhai J, Wan M, Jiang L. Chemical dualresponsive wettability of superhydrophobic PANI-PAN coaxial nanofibers. Macromol Rapid Commun. 2007;28(10):1135-1141.

26. Li SF, Wu WT. Lipase-immobilized electrospun PAN nanofibrous membranes for soybean oil hydrolysis. Biochem Eng J. 2009;45(1):48-53.

27. Li S-F, Fan Y-H, Hu R-F, Wu W-T. Pseudomonas cepacia lipase immobilized onto the electrospun pan nanofibrous membranes for biodiesel production from soybean oil. J Mol Catal B Enzym. 2011;72(1-2): $40-45$.

28. Wu X-M, Branford-White CJ, Yu D-G, Chatterton NP, Zhu L-M. Preparation of core-shell pan nanofibers encapsulated $\alpha$-tocopherol acetate and ascorbic acid 2-phosphate for photoprotection. Colloids Surf B Biointerfaces. 2011;82(1):247-252. 
29. YalcinkayaF, YalcinkayaB,PazourekA, MullerovaJ,StuchlikM, MaryskaJ Surface modification of electrospun PVDF/PAN nanofibrous layers by low vacuum plasma treatment. Int J Polymer Sci. 2016;2016(3):1-9.

30. Ren S, Dong L, Zhang X, et al. Electrospun nanofibers made of silver nanoparticles, cellulose nanocrystals, and polyacrylonitrile as substrates for surface-enhanced Raman scattering. Materials. 2017;10(1):68.

31. Wang S, Hu F, Li J, et al. Design of electrospun nanofibrous mats for osteogenic differentiation of mesenchymal stem cells. Nanomedicine. 2018;14(7):2505-2520.

32. Ye C, Zhao J, Zheng Y, et al. Preparation of Poly(lactic-co-glycolic acid)-based composite microfibers for postoperative treatment of tumor in NIR I and NIR II biowindows. Macromol Biosci. 2018;18(10): 1800206-1800213.

33. Khan MQ, Kharaghani D, Nishat N, et al. In vitro assessment of dualnetwork electrospun tubes from poly(1,4 cyclohexane dimethylene isosorbide terephthalate)/PVA hydrogel for blood vessel application. J Appl Polym Sci. 2018;47222.
34. Khan M, Kharaghani D, Ullah S, et al. Self-Cleaning properties of electrospun PVA/TiO2 and PVA/ZnO nanofibers composites. Nanomaterials. 2018;8(9):644.

35. Haider A, Haider S, Kang IK. A comprehensive review summarizing the effect of electrospinning parameters and potential applications of nanofibers in biomedical and biotechnology. Arab J Chem. 2018;11(8):1165-1188.

36. Khajavi R, Abbasipour M. Electrospinning as a versatile method for fabricating coreshell, hollow and porous nanofibers. Scientia Iranica. 2012;19(6):2029-2034.

37. Pan T, Wang W. From cleanroom to desktop: emerging micronanofabrication technology for biomedical applications. Ann Biomed Eng. 2011;39(2):600-620.

38. Gunn J, Zhang M. Polyblend nanofibers for biomedical applications: perspectives and challenges. Trends Biotechnol. 2010;28(4):189-197.

39. Xie J, Li X, Xia Y. Putting electrospun nanofibers to work for biomedical research. Macromol Rapid Commun. 2008;29(22):1775-1792.
International Journal of Nanomedicine

\section{Publish your work in this journal}

The International Journal of Nanomedicine is an international, peerreviewed journal focusing on the application of nanotechnology in diagnostics, therapeutics, and drug delivery systems throughout the biomedical field. This journal is indexed on PubMed Central, MedLine, CAS, SciSearch $®$, Current Contents $\AA /$ Clinical Medicine,

\section{Dovepress}

Journal Citation Reports/Science Edition, EMBase, Scopus and the Elsevier Bibliographic databases. The manuscript management system is completely online and includes a very quick and fair peer-review system, which is all easy to use. Visit http://www.dovepress.com/ testimonials.php to read real quotes from published authors.

Submit your manuscript here: http://www.dovepress.com/international-journal-of-nanomedicine-journal 